\title{
RELACIONES GENÓMICAS ENTRE DOS ESPECIES HEXAPLOIDES DE TURNERA, T. ORIENTALIS Y T. VELUTINA, Y UNA DIPLOIDE, T. GRANDIFLORA (TURNERACEAE, SERIE TURNERA)
}

\author{
por AVELIANO FERNÁNDEZ ${ }^{1}$ y MARÍA MERCEDES ARBO 2
}

\begin{abstract}
Summary
We hybridized two hexaploid species $(2 n=6 x=30)$ T. orientalis and T. velutina and one diploid species $(2 n=2 x=10), \quad$. grandiflora. These species had regular meiosis forming 15 II the hexaploids and $5 \|$ the diploid. One hexaploid, $2 n=6 x=30$ (T. orientalis $x T$. velutina), one pentaploid, $2 n=5 x=25$ (T. orientalis $x$ T. grandiflora) and one tetraploid, $2 n=4 x=20$ (T. velutina $x T$. grandiflora) hybrids were obtained and studied cytologically to determine their genomic relationships. Meiotic behavior in the $T$. orientalis $\times T$. velutina hybrid was very irregular, with many laggard chromosomes and bridges at $\mathrm{Al}$ and All. Mean pairing relationships were 10,38 I, 9,66 II, 0,07 III and 0,03 IV. The hybrid T. orientalisx T. grandiflora presented irregular meiosis. Mean pairing relationships were $14,66 \mathrm{I}$ and 5,16 I. T. velutina $\times$ T. grandiflora presented the most irregular meiosis, with mean pairing relationships of 6,94 I, 6,42 II and 0,05 IV. The three hybrids were sterile. The genome constitution of $T$. orientalis is $\mathrm{A}^{\circ} \mathrm{A}^{\circ} \mathrm{BBB} \mathrm{B}^{\circ} \mathrm{B}^{\circ}$ and of $T$. grandiflora is $\mathrm{C}^{9} \mathrm{C}^{9}$. On the basis of chromosome associations in the hybrids, we propose the genomic formula $A A A^{v} A^{v} C^{v} C^{v}$ for $T$. velutina. One genome of $T$. orientalis is similar to one of the three genomes of $T$. velutina. The latter species shows one genome similar to the genome of $T$. grandiflora. Both $T$. orientalis and $T$. velutina are segmentary alohexaploids.
\end{abstract}

Key words: Turnera, genomic relationships, chromosome pairing, segmentary alohexaploids, genomic formula

Palabras claves: Turnera, relaciones genómicas, apareamiento cromosómico, alohexaploides segmentarios, fórmulas genómicas

Introducción

Turnera L. está dividido en nueve series (Urban, 1883), que poseen tres números bási$\cos , \mathrm{x}=5, \mathrm{x}=7$ y $\mathrm{x}=13$. Turnera (=Canaligerae) es la única serie con número básico $x=5$ y sus niveles de ploidía varían desde diploides hasta octoploides (Fernández, 1987). Desde 1982 rea-

\footnotetext{
${ }^{1}$ Facultad de Ciencias Exactas y Naturales y Agrimensura.

${ }^{2}$ Facultad de Ciencias Agrarias

1,2 Miembros de la Carrera del Investigador Científico del CONICET. Instituto de Botánica del Nordeste (UNNECONICET), Casilla de Correo 209, 3400 Corrientes, Argentina.
}

lizamos cruzamientos controlados (Arbo y Fernández, 1987) para obtener híbridos interespecíficos con la finalidad de determinar las relaciones genómicas entre las especies involucradas. Como resultados de los estudios citogenéticos publicamos varios trabajos, en los que dimos conocer las fórmulas genómicas de ocho especies diploides, una tetraploide, una hexaploide y una octoploide (Fernández, 1997, Fernández y Arbo, 1989, 1990, 1993a, 1993b, 1996), entre las cuales figuran las de $T$. grandiflora (Urb.) Arbo y de T. orientalis (Urb.) Arbo.

En el presente trabajo se realiza el análisis citogenético de los híbridos entre $T$. orientalis 
$(2 n=6 x=30), \quad T$. velutina $(2 n=6 x=30)$ y $T$. grandiflora $(2 n=2 x=10)$, con el fin de determinar sus relaciones filogenéticas y establecer la fórmula genómica de T. velutina Presl.

\section{Material y métodos}

Los progenitores se indican con la sigla utilizada en el invernáculo. Los ejemplares testigo de progenitores e híbridos se conservan en el herbario del Instituto de Botánica del Nordeste (CTES).

T. orientalis (O). Arbo 1538. Argentina, Corrientes.

T. velutina (Ve). Koch y Fryxell 78341, México, Oaxaca, Santo Domingo.

T. grandiflora (G2). Fernández 366, Argentina, Corrientes, Paso de la Patria.

$\mathrm{O} \times \mathrm{Ve}$, cultivado en Corrientes (CTES 222928)

O x G2, cultivado en Corrientes (CTES 91737)

Ve x G2, cultivado en Corrientes (CTES 219575)

El procedimiento usado para los cruzamientos está descripto en Arbo y Fernández (1987) e incluye los siguientes pasos: castración de las flores de las plantas madres, polinización con anteras de la planta seleccionada como padre, marcación de la flor madre indicando en el rótulo el progenitor masculino. Las semillas obtenidas se sembraron en macetas individuales y los híbridos se trasplantaron después de desarrollar el primer par de hojas.

Las preparaciones para el estudio de la meiosis se hicieron fijando los botones florales con cinco partes de etanol absoluto y una parte de ácido láctico (Fernández, 1973) y coloreando con la técnica de Feulgen. Para mitosis se siguió el mismo procedimiento, previo pretratamiento de las raíces con 8-hidroxiquinoleína 0,002M durante 3 horas a temperatura de laboratorio, entre 18 y $26^{\circ} \mathrm{C}$. Para estimar el porcentaje de fertilidad de polen se usó la técnica de coloración con carmín-glicerina, contando no menos de 300 granos por flor.

\section{Morfología y polen de progenitores e híbridos}

Los caracteres morfológicos diferenciales de $T$. orientalis y $T$. grandiflora se describieron en trabajos previos (Fernández y Arbo 1993a y 1996): la primera especie presenta flores amarillas homostilas, mientras la segunda tiene flores blanco-violáceas heterostilas. El polen de $T$. orientalis es reticulado, con aréolas pequeñas y escasas báculas libres, mientras el de $T$. grandiflora presenta retículo de mallas amplias, con numerosas báculas libres.

T. velutina es un arbusto con follaje verde tendiente a glauco, con pelos simples de laxos a muy densos, a veces amarillentos. Las hojas aserradas tienen forma variable, ovada, elíptica, latielíptica, angustiovada, a veces obovada. Sus flores son homostilas, de 30-50 mm diám., pétalos de color amarillo muy pálido, con base amarilla, y anteras y polen amarillos. El gineceo es notoriamente más largo que el androceo, hasta $7 \mathrm{~mm}$, y los estigmas presentan 24-48 ramas. El tamaño de los granos de polen tiene una media de 71,07 $\mu \mathrm{m}$. La antesis se inicia alrededor de las 8,45 hs. Las plantas son autocompatibles.

La fertilidad promedio del polen fue cercana al $100 \%$ en los tres progenitores.

El híbrido $T$. orientalis $x T$. velutina tiene hojas de forma variable, de color similar al de $T$. velutina. Las flores son homostilas, de aproximadamente $30 \mathrm{~mm}$ de diámetro, pétalos amarillo pálido. Las anteras permanecen cerradas y el gineceo es 2,5-4,5 mm más largo que el androceo. La fertilidad promedio del polen es de $2,8 \%$.

El híbrido T. orientalis $x T$. grandiflora presenta hojas ovadas, de haz lisstrosa, con olor desagradable al ser estrujadas, heredada de $T$. grandiflora. Las flores son longistilas, de $32 \mathrm{~mm}$ de diámetro, los pétalos son amarillos, con mancha basal violácea de borde superior esfumado y venas violáceas. Las anteras son anaranjadas, los estilos son erguidos, conniventes y los estigmas multífidos. La fertilidad promedio del polen es de 3,92\%.

El híbrido $T$. velutina $x T$. grandiflora posee follaje de color similar al de T. velutina. Las hojas aserradas son generalmente elípticas, de base cuneada y ápice agudo. Las flores son marcadamente longistilas, tienen aproximadamente $50 \mathrm{~mm}$ de diámetro, los pétalos de color marfil presentan una mancha basal obtriangtular violeta, orlada de amarillo. Las anteras son de color amarillo vivo, igual que los estigmas. El 
gineceo es 8-9 mm más largo que el androceo. La fertilidad promedio del polen es de $1 \%$.

\section{Resultados y discusión}

Los progenitores presentaron meiosis regular, formando regularmente $15 \mathrm{II}$ en $T$, orientalis y $T$. velutina y 5 II en $T$, grandiflora.

De los cruzamientos realizados se obtuvieron los híbridos: $T$. orientalis $\times T$. velutina,
$2 \mathrm{n}=6 \mathrm{x}=30 ; \quad$ T. orientalis $\mathrm{x}$ T. grandiflora, $2 \mathrm{n}=5 \mathrm{x}=25$ y $T$. velutina $\times \mathrm{T}$. grandiflora, $2 \mathrm{n}=4 \mathrm{x}=20$.

En el híbrido $T$. orientalis $\times T$. velutina, $2 n=6 x=30$, se analizaron 26 CMP y se encontraron nueve configuraciones meióticas diferentes (Tabla 1), siendo la más frecuente $10 \mathrm{I}+$ 10 II en $26,93 \%$ de las CMP, en la Fig. 1A se observan 4 I + 10 II + 2 III. El promedio de univalentes fue de 10,38, el de bivalentes fue

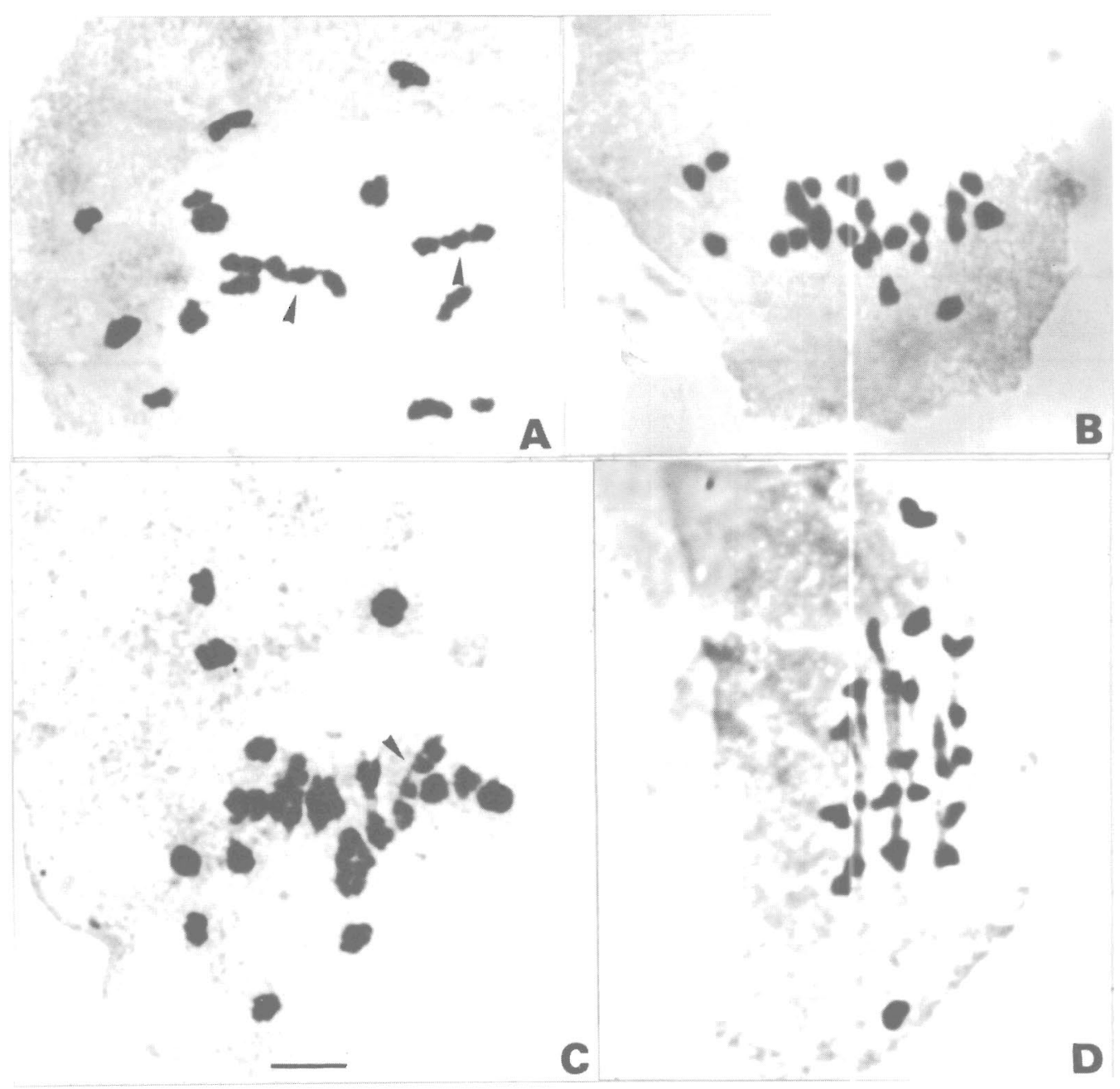

Fig. 1. Cromosomas meióticos en los híbridos. A, T. orientalis $x$ T. velutina, $2 n=30$, metafase I, 4 I +10 II +2 III. Las flechas señalan trivalentes. B y C, T. orientalis x T. grandiflora; $2 \mathrm{n}=25, \mathrm{~B}$, metafase I, $15 \mathrm{I}+5 \mathrm{II}, \mathrm{C}$, inicio de anafase I, la flecha señala puente en $\mathrm{E}$, producto de intercambio tipo U proximal. D, T. velutina $\times$ T. grandiflora, $2 \mathrm{n}=20$, inicio de anafase I, $10 \mathrm{I}+5 \mathrm{II}$. Escala $=5 \mu \mathrm{m}$. 
de 9,61 , el de trivalentes fue de 0,07 y el de cuadrivalentes fue de 0,03 (Tabla 2).

En T. orientalis $x T$. grandiflora, $2 \mathrm{n}=5 \mathrm{x}=25$, se analizaron $42 \mathrm{CMP}$, encontrándose seis configuraciones meióticas diferentes (Tabla 1), siendo la más frecuente 15 I + 5 II (Fig. 1B) en 57,14 $\%$ de las CMP. El promedio de univalentes fue de 14,66 y el de bivalentes fue de 5,16 (Tabla 2).

En $T$. velutina $\times T$. grandiflora, $2 \mathrm{n}=4 \mathrm{x}=20$, se analizaron 19 CMP y se encontraron siete configuraciones meióticas diferentes (Tabla 1), siendo la más frecuente 8 I + 6 II en 26,32 \% de las CMP; en la Fig. 1B se observan 10 I + 5 II. El promedio de univalentes fue de 6,94, el de bivalentes fue de 6,42 y el de cuadrivalentes fue de 0,05 (Tabla 2). En una célula se observó un bivalente que se interpreta como intercambio tipo-U (Fig. 1C).
Los tres híbridos presentaron cromosomas rezagados y puentes en anafase II y anafase II. La Fig. 2A muestra una célula de T. orientalis $x$ T. velutina en telofase $I$, donde se observan cromosomas que han pasado por citomixis y cuatro univalentes rezagados que se están dividiendo ecuacionalmente. Estos cromosomas rezagados finalmente llegan a los polos telofásicos, como se observa en la Fig. 2B, que muestra una célula en prometafase II. En ella se observan cromosomas con sus dos cromátidas y otros con una sola cromátida; estos últimos serían los univalentes que se dividieron ecuacionalmente en anafase I.

En $T$. velutina $\times$ T. grandiflora se han observado puentes sin fragmentos (Fig. 2C) y con fragmentos en T. orientalis X T. grandiflora (Fig. 2D).

Tabla 1. Configuraciones meióticas en metafase I observadas en los hibridos estudiados

\begin{tabular}{|c|c|c|c|c|c|c|c|c|}
\hline $\begin{array}{c}O \times V e \\
(2 n=30)\end{array}$ & frec. & $\%$ & $\begin{array}{c}O \times G 2 \\
(2 n=25)\end{array}$ & frec. & $\%$ & $\begin{array}{c}V \times G 2 \\
(2 n=20)\end{array}$ & frec. & $\%$ \\
\hline $20 \mathrm{I}+5 \mathrm{II}$ & 1 & 3,84 & 19 I + 3 II & 2 & 4,76 & $12 \mathrm{I}+4 \mathrm{II}$ & 2 & 10,52 \\
\hline $16 \mathrm{I}+7 \mathrm{II}$ & 2 & 7,69 & $17 \mathrm{I}+4 \mathrm{II}$ & 5 & 11,90 & $0 \mathrm{I}+5 \mathrm{II}$ & 2 & 10,52 \\
\hline $14 \mathrm{I}+8 \mathrm{II}$ & 5 & 19,22 & $15 I+5 I I$ & 24 & 57,14 & $8 \mathrm{I}+6 \mathrm{II}$ & 5 & 26,32 \\
\hline 12 I + 9 II & 1 & 3,84 & $13 I+6 I I$ & 8 & 19,06 & $6 \mathrm{I}+7 \mathrm{II}$ & 4 & 21,06 \\
\hline $10 \mathrm{I}+10 \mathrm{II}$ & 7 & 26,93 & $11 \mathrm{I}+7 \mathrm{II}$ & 2 & 4,76 & $4 I+8 I I$ & 4 & 21,06 \\
\hline $9 \mathrm{I}+7 \mathrm{II}+1 \mathrm{III}+1 \mathrm{IV}$ & 1 & 3,84 & $7 \mathrm{I}+9 \mathrm{II}$ & 1 & 2,38 & $2 \mathrm{I}+9 \mathrm{II}$ & 1 & 5,26 \\
\hline $8 I+11 \mathrm{II}$ & 4 & 15,40 & & & & $6 I+5 I I+1 I V$ & 1 & 5,26 \\
\hline $6 \mathrm{I}+12 \mathrm{II}$ & 4 & 15,40 & & & & & & \\
\hline $4 \mathrm{I}+10 \mathrm{II}+2 \mathrm{III}$ & 1 & 3,84 & & & & & & \\
\hline
\end{tabular}

Tabla 2. Promedio \pm E.S. y variación de las asociaciones cromosómicas en metafase I

\begin{tabular}{|c|c|c|c|c|c|c|c|}
\hline Híbrido & $2 n$ & I & II & III & IV & $\mathrm{N}^{\circ}$ cél. & Fert. \% \\
\hline \multicolumn{8}{|l|}{$T$ : orientalis } \\
\hline $\begin{array}{c}\mathrm{x} \\
\text { T. velutina }\end{array}$ & 30 & $\begin{array}{c}10,38 \pm 0,63 \\
6-14\end{array}$ & $\begin{array}{c}9,61 \pm 0,31 \\
8-12\end{array}$ & $0,07 \pm 0,05$ & $0,03 \pm 0,03$ & 26 & 2,80 \\
\hline \multicolumn{8}{|l|}{$\mathrm{T}$. orientalis } \\
\hline$\stackrel{\mathrm{x}}{\text { T. grandiflora }}$ & 25 & $\begin{array}{c}14,66 \pm 0,31 \\
7-19\end{array}$ & $\begin{array}{c}5,16 \pm 0,15 \\
3-9\end{array}$ & & & 42 & 3,00 \\
\hline \multicolumn{8}{|l|}{ T. velutina } \\
\hline$\stackrel{x}{\quad}$ & 20 & $\begin{array}{c}6,94 \pm 0,63 \\
2-12\end{array}$ & $\begin{array}{c}6,42 \pm 0,32 \\
4-9\end{array}$ & $0-1$ & $0,05 \pm 0,05$ & 19 & 1,00 \\
\hline
\end{tabular}



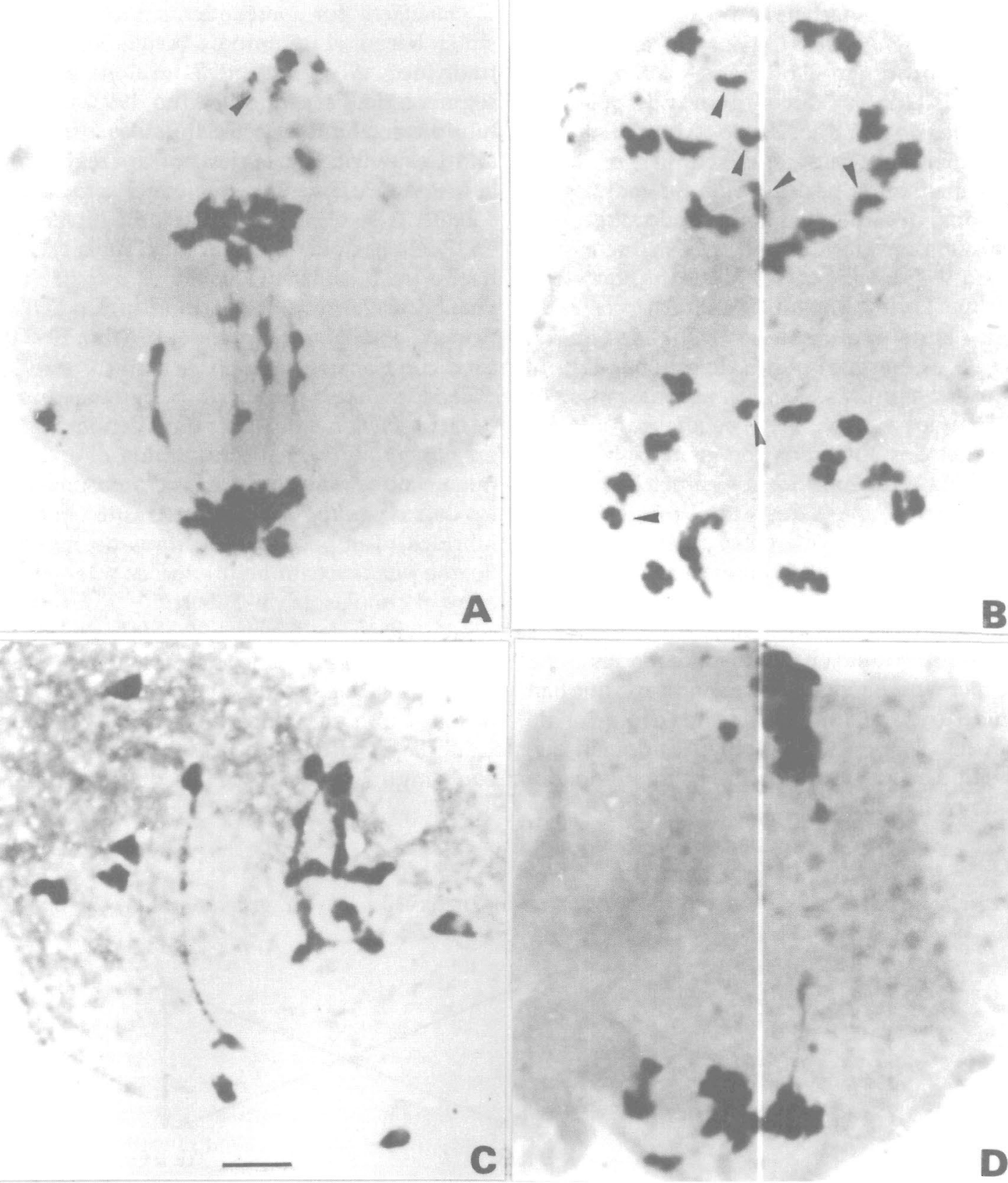

C

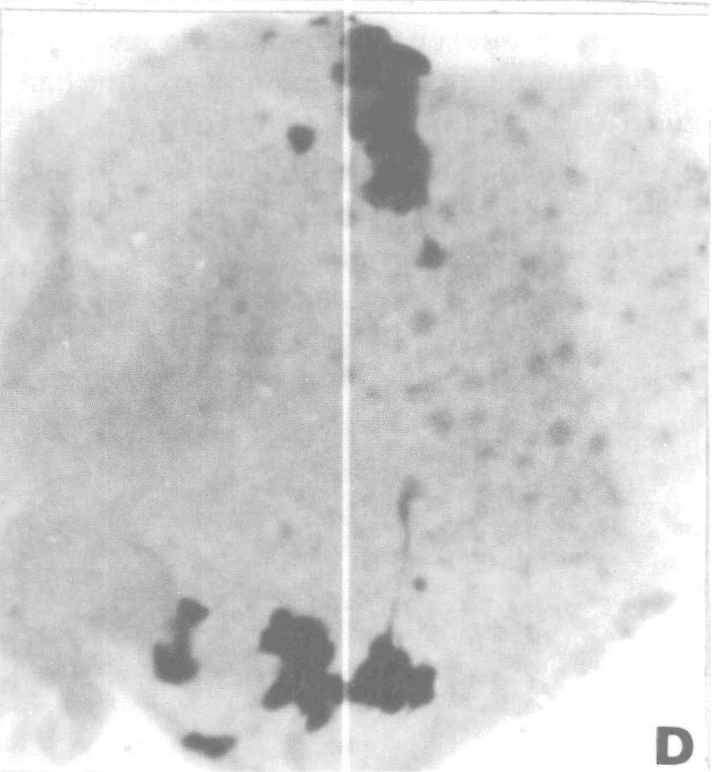

Fig. 2. Cromosomas meióticos de los híbridos. A y B,T. orientalis $x$ T. velutina, $2 n=30, A$, telofase $I_{1} 4$ univalentes rezagados dividiéndose ecuacionalmente, la flecha indica cromosomas que han pasado por citomixis; B, prometafase II, las flechas indican cromosomas con una sola cromátida. C, T. velutina $\times$ T. grandiflora, $2 \mathrm{n}=20$, anafase I con puentes sin fragmentos. D, T. orientalis $\times$ T. grandiflora, $2 \mathrm{n}=25$, metafase II con puente remanente y fragmento. 
El único híbrido viable $T$. orientalis $\times T$. grandiflora $(2 n=5 x=25)$, se obtuvo después de varios años de cruzamientos mediante gametos no reducidos de uno de los progenitores. El número cromosómico del híbrido indica que en el cruzamiento participaron gametos no reducidos de T. grandiflora $(2 n=2 x=10)$, es decir que sólo se produjeron híbridos viables con $T$. orientalis $(2 n=6 x=30)$ con granos de polen resultantes de una división no reduccional. Anteriormente se obtuvieron semillas, presumiblemente con la participación de gametos reducidos, que dieron plántulas cloróticas, las cuales no progresaron más allá de ese estado (Arbo y Fernández, 1987; Fernández y Arbo 1993a). En los cruzamientos recíprocos realizados hasta el presente los resultados fueron negativos, hasta el punto de no obtenerse ni siquiera semillas. En un trabajo anterior (Fernández y Arbo, 1990) se analizaron otros dos híbridos obtenidos con gametos no reducidos. En estos híbridos los gametos no reducidos fueron aportados por el progenitor femenino, mientras que en el presente caso ( $T$. orientalis $\mathrm{x} T$. grandiflora) fueron aportados por el progenitor masculino (T. grandiflora). Con ésto se prueba que los gametos no reducidos se producen tanto en macrosporogénesis como en microsporogénesis, lo cual indicaría que han tenido un papel importante en la evolución de Turnera. Harlan y de Wet (1975) opinan que este tipo de gametos tiene un papel preponderante en la poliploidización.
Otro hecho digno de mención son las asociaciones de los cromosomas en $T$. orientalis $x$ T. grandiflora. En este híbrido se esperaba encontrar en todas las células, como mínimo, 5 II, que corresponderían a los cromosomas de T. grandiflora por apareamiento autosindético; sin embargo, el mínimo de bivalentes encontrado fue 3. Al ser T. orientalis un alohexaploide segmentario (Fernández y Arbo, 1993a), en los híbridos con otras especies diploides forma con alta frecuencia bivalentes por apareamiento autosindético, mientras que con T. grandiflora solamente se observaron más de 5 bivalentes en 12 células de las 42 estudiadas (Tabla 1). Este hecho ya fue observado en $T$. aurelii $\times T$. grandiflora $(2 \mathrm{n}=5 \mathrm{x}=25)$, otro híbrido donde participa T. grandiflora (Fernández y Arbo, 1993a). En dicho trabajo, tratando de explicar este fenómeno se había propuesto la siguiente hipótesis: probablemente exista en el genoma de $T$. grandiflora algún gen que impida el apareamiento autosindético entre los cromosomas de los otros genomios involucrados en los híbridos. Tampoco se descarta la posibilidad de que pueda existir algún tipo de interacción entre el citoplasma de $T$. aurelii y $T$. orientalis con el núcleo de T. grandiflora, que impida el apareamiento autosindético, inclusive entre los mismos cromosomas de T. grandiflora. Cabe destacar que en ambos híbridos el genomio $\mathrm{C}^{\mathrm{g}}$ de T. grandiflora no presenta homeología con ningún genomio de los otros progenitores.
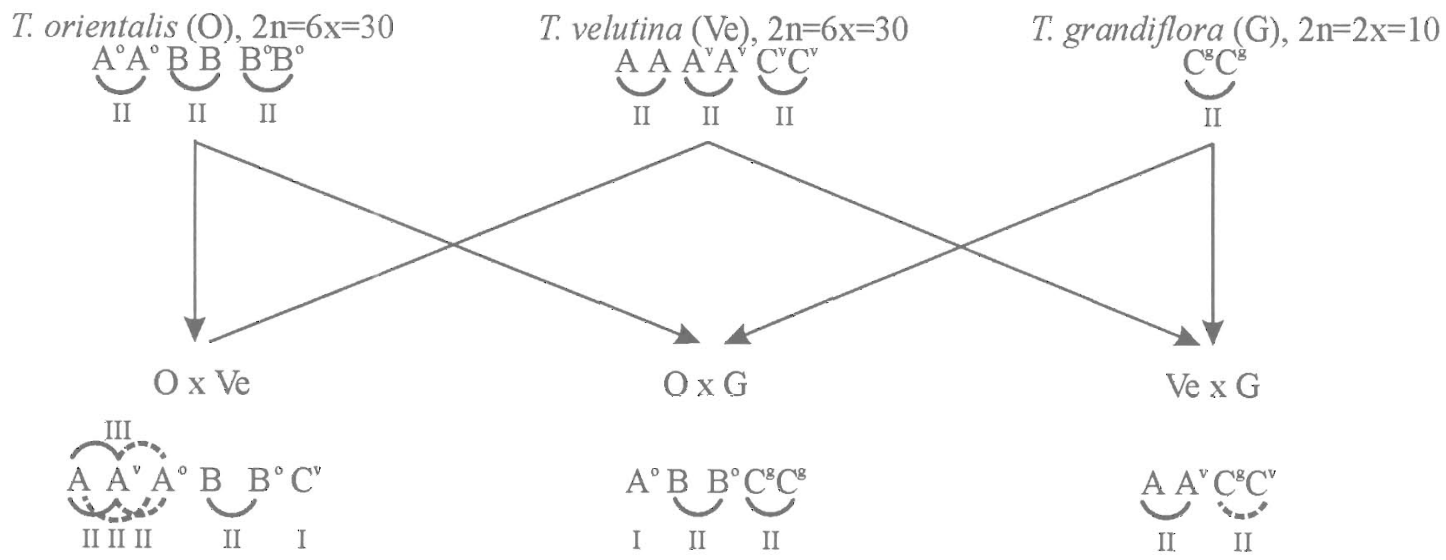

Fig. 3. Fórmulas genómicas y asociaciones cromosómicas en los progenitores e híbridos. A pareamiento autosindético

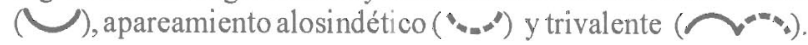


Una situación diferente es lo observado en $T$. velutina $\times T$. grandiflora donde el porcentaje de bivalentes observado es elevado; se han encontrado células con 9 II, o sea que se ha producido apareamiento autosindético entre los cromosomas de $T$. velutina y alosindético entre los cromosomas de ambas especies. Este hecho se debería a que el genomio de $T$. grandiflora y uno de los de T. velutina son homeólogos. T. velutina es una especie endémica de México, donde también se encuentra T. caerulea. El genomio de esta última especie, que podría ser una de las que participaron en el origen de $T$. velutina, es homeólogo al de T. grandiflora (Fernández y Arbo 1996).

\section{Conclusiones}

En trabajos previos se determinó la fórmula genómica $\mathrm{A}^{\circ} \mathrm{A}^{\circ} \mathrm{BBB}^{\circ} \mathrm{B}^{\circ}$ para $T$. orientalis y $\mathrm{C}^{8} \mathrm{C}^{8}$ para T. grandiflora. En este trabajo por las asociaciones cromosómicas y configuraciones halladas en los híbridos, se propone la fórmula genómica $\mathrm{AAA}^{\mathrm{v}} \mathrm{A}^{\mathrm{v}} \mathrm{C}^{\mathrm{v}} \mathrm{C}^{\mathrm{v}}$ para $T$. velutina.

En la Fig. 3 se muestran las fórmulas genómicas y las asociaciones cromosómicas de los progenitores e híbridos. En T. orientalis $\times T$. velutina los bivalentes observados se formarían por apareamiento autosindético entre los cromosomas de los genomios $\mathrm{AA}^{\mathrm{v}}$ de T. velutina y $B^{\circ}$ de $T$. orientalis, también por apareamiento alosindético entre los cromosomas de los genomios $\mathrm{AA}^{\circ}$ y $\mathrm{A}^{\circ} \mathrm{A}^{\mathrm{v}}$ de ambas especies. Los trivalentes se podrían formar por apareamiento autosindético y alosindético al mismo tiempo, es decir entre los cromosomas de los tres genomios A de ambas especies. Entre los univalentes se encontrarían los cinco cromosomas de $T$. velutina del genomio $\mathrm{C}^{\mathrm{v}}$.

En $T$. orientalis $x T$. grandiflora los bivalentes se formarían únicamente por apareamiento autosindético entre los cromosomas de los genomios $\mathrm{BB}^{\circ}$ de $T$. orientalis ${ }^{\circ} \mathrm{C}^{\mathrm{B}} \mathrm{C}^{\mathrm{B}}$ de $T$. grandiflora. Entre los univalentes se encontra- rían los cinco cromosomas de $T$. orientalis del genomio $\mathrm{A}^{\circ}$.

En $T$. velutina $\times T$. grandiflora los bivalentes se formarían por apareamiento autosindético entre los cromosomas de los genomios $\mathrm{AA}^{\mathrm{v}}$ de T. velutina y alosindético entre los cromosomas de los genomios $\mathrm{C}^{\mathrm{g}} \mathrm{C}^{\mathrm{r}}$ de ambas especies.

La presencia del cuadrivalente en los híbridos $T$. orientalis $\times T$. velutina y $T$. velutina $x$ T. grandiflora se debería a la existencia de translocaciones entre los cromosomas de los genomios $\mathrm{A}^{v} \mathrm{y}^{v} \mathrm{de} T$. velutina. Se presume esta hipótesis debido a que esta especie aportó ambos genomios a los dos híbridos, donde se encontró esa asociación cromosómica.

\section{Bibliografía}

ARBO, M.M. y A. FERNÁNDEZ. 1987. Cruzamientos intra e interespecíficos en Turnera, serie Canaligerae. Bonplandia 6(1): 23-38.

FERNÁNDEZ, A. 1973. El ácido láctico como fijador cromosómico. Bol. Soc. Argent. Bot. 15(2-3): 287-290.

— 1987. Estudios cromosómicos en Turnera y Piriqueta (Turneraceae). Bonplandia 6(1): 1-21.

—1997. Estudio citogenético en híbridos entre una especieoctoploide, Turneraaurelii y dos diploides, T.caerulea y T. joelii. Bonplandia 9(3-4): 281-286.

FERNÁNDEZ, A. y M.M. ARBO. 1989. Relaciones genómicas entre cuatro especies diploides de 'l'urnera con flores amarillas (Serie Canaligerae). Bonplandia 6(2): 93-109.

— 1990. Gametas no reducidas y relaciones genómicas en tres especies de Turnera (Turneraceae). Darwiniana 30(1-4): 21-26.

— 1993a. Relaciones genómicas entre seis especies de Turnera (Serie Canaligerae) del Paraguay. Candollea 48: 305-318.

— 1993b. Citogenética de híbridos entre Turnera grandidentata $(4 x)$ y $T$. subulata y $T$. scabra $(2 x)$ (Tumeraceae). Bonplandia 7(1-4): 119-127.

— 1996. Relaciones genómicas entre las especies diploides de flores blanco-azuladas de Turnera (Serie Canaligerae). Bonplandia 9(1-2): 95-102.

HARLAN, J.R. \& J.M.J. de WET. 1975. On Ö. Winge and a prayer: the origins of polyploidy. Bot. Rev. 41(4): 361390.

URBAN, I. 1883. Monographie der familie der Turneraceen. Jahrb. Königl. Bot. Gart. Berlin 2: 1-152. 